\title{
Estimation of SH-Wave Amplification in the Bandung Basin Using Haskell's Method
}

\author{
Afnimar \\ Laboratorium Seismology, Faculty of Mining and Petroleum Engineering, \\ Institut Teknologi Bandung, Jalan Ganesha No 10, Bandung 40132, Indonesia \\ Email: afnimar@geoph.itb.ac.id
}

\begin{abstract}
The Bandung basin is a large basin in Indonesia surrounded by mountains that are associated with faults. There is the possibility of earthquakes generated by these faults shaking populated areas in the basin. The consequences will be worse because the shaking is amplified by the sediment layer of the basin. We have estimated the amplification of SH-waves generated by the Lembang fault using Haskell's method for multilayer models. The pattern of amplification is a decreasing value with increasing distance from the Lembang fault. This pattern is valid for low-frequency incident waves. For higherfrequency incident waves, the pattern looks more complicated. Fortunately, there are many areas with low amplification values. Hopefully, this result will help the local government in making decisions regarding construction planning in this region. Of course, the final objective is to reduce earthquake risks.
\end{abstract}

Keywords: amplification factor; basin sediment structure; earthquake mitigation; frequency and incidence angle dependence; Haskell's method.

\section{$1 \quad$ Introduction}

Motivated by the high seismicity of the Indonesian region, Japan and Indonesia have set up made a research collaboration to mitigate this natural hazard. The title of the research is "Multi-disciplinary Hazard Reduction from Earthquakes and Volcanoes in Indonesia". One of the research topics is the prediction of strong motion in the Bandung basin area (as shown in Figure 1) that can be generated by the Lembang fault slip. This prediction must be supported by estimation of fault and sediment structure models. The fault model was estimated by the empirical formulation of Irikura and Miyake [1], while the sediment structure was determined by use of microtremor array observation. In this research, we have calculated the synthetic amplification factor of SHwaves, which can later be used for strong motion prediction. We applied SHwaves instead of P-SV-waves because shaking is dominant in the horizontal component.

The prediction calculation was based on Haskell's method [2] for multilayer models. Haskell applied propagator matrices to calculate the wave amplitudes

Received March $7^{\text {th }}, 2013$, Revised October $11^{\text {th }}, 2013$, Accepted for publication January $18^{\text {th }}, 2014$

Copyright (C) 2014 Published by ITB Journal Publisher, ISSN: 2337-5779, DOI: 10.5614/j.eng.technol.sci.2014.46.1.6 
from the basement to the surface. We applied it to a half-space model at each point on the surface and analyzed the influence of incidence angle, frequency and sediment structure.

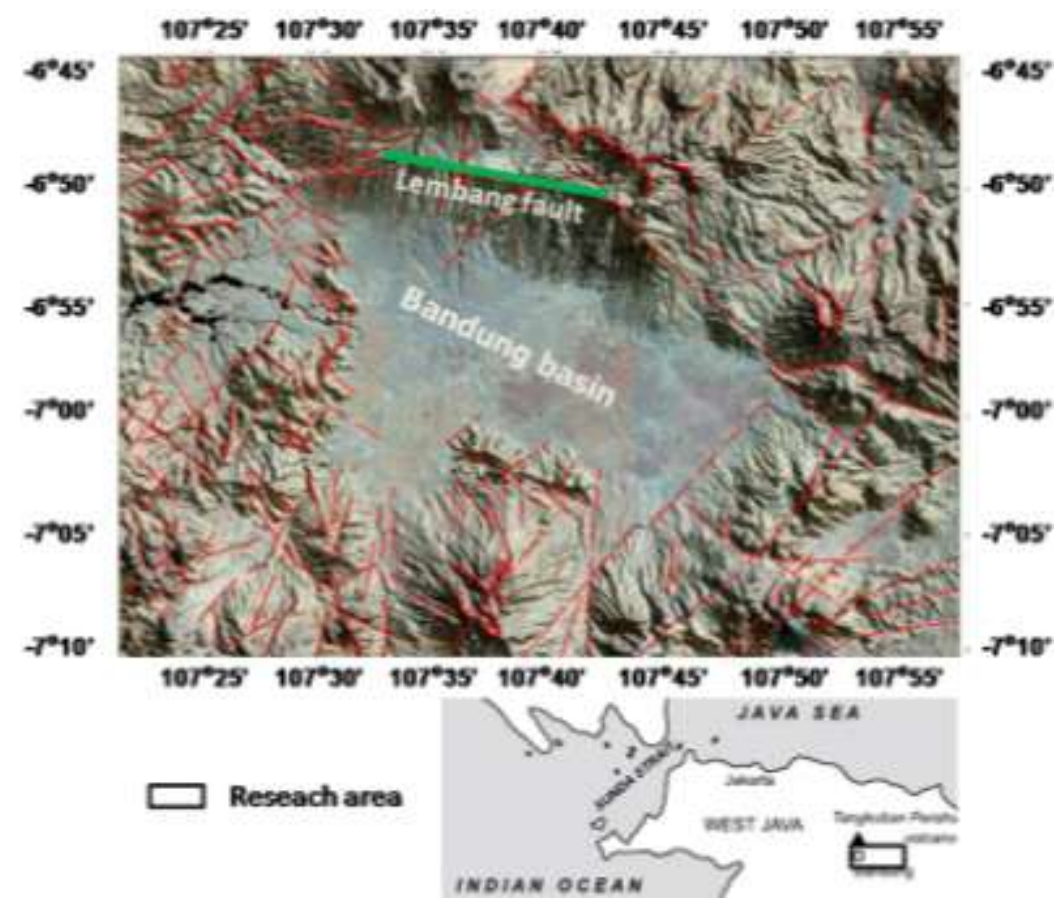

Figure 1 Location of the research area in the West Java province. The Lembang fault (green line) is located north of the Bandung basin. The fault lines (red line) were identified by Lumbanbatu [3].

\section{$2 \quad$ Planar SH-Waves in Layered Media}

Thompson [4] and Haskell [5] describe a method for solving the problem of a harmonic planar P-, SV-, or SH-wave through a vertically stratified medium. This is known as the Thompson-Haskell propagator matrix method and is the basis of a widely used computer program. The formulation of Haskell's method is as follows [2]. Consider a stack of $n-1$ plane layers over an elastic half-space with a planar SH-wave incident from the half-space. Although we consider the plane wave to be harmonic, we can sum the harmonic solutions to form the solution for any time history of the incident wave. The displacement solution $\vec{u}$ over the $\left(x_{1} x_{3}\right)$ half-space is of the form

$$
\vec{u}=f\left(x_{3}\right) \cos \left[\omega\left(t-p x_{1}\right)-\delta\right] \hat{e}_{2},
$$


where $f\left(x_{3}\right)$ is a function of depth that allows us to match the appropriate boundary conditions, $\omega$ is radian frequency, $t$ is time, $p$ is ray parameter, $\delta$ is a phase shift that may also be a function of depth, and $\hat{e}_{2}$ is a unit vector of the SH-wave oscillation direction.

At each boundary, there are two important conditions: continuous displacement and stress. Thompson and Haskell devised a clever solution method that writes these boundary conditions in matrix form. If we denote the amplitude of the upgoing SH-wave incident at the bottom of the stack as $f^{I}$, then the amplitude at the earth's surface $f^{0}$ can be written as

$$
f^{0}=f^{I}\left(\frac{2 \mu_{n} \gamma_{n}}{\mu_{n} \gamma_{n} A_{11}+A_{21}}\right)
$$

where

$$
\begin{aligned}
& \gamma_{n}=\sqrt{\frac{c^{2}}{\beta_{n}}-1}, \\
& \mathbf{A}=\prod_{m=n-1}^{1} \mathbf{a}_{m}=\mathbf{a}_{n-1} \mathbf{a}_{n-2} \mathbf{a}_{n-3} \cdots \mathbf{a}_{1}, \\
& \mathbf{a}_{m}=\left[\begin{array}{cc}
\cos Q_{m} & i\left(\mu_{m} \gamma_{m}\right)^{-1} \sin Q_{m} \\
i\left(\mu_{m} \gamma_{m}\right)^{-1} \sin Q_{m} & \cos Q_{m}
\end{array}\right]
\end{aligned}
$$

and

$$
Q_{m}=\frac{\omega h_{m} \gamma_{m}}{c} .
$$

The variables in the above equations can be explained as follows: $\mu$ is shear modulus, $c$ is horizontal phase velocity, $\beta$ is $\mathbf{S}$-wave velocity, $\mathbf{A}$ is product of reflection and transmission, $i$ is an imaginary number and $h$ is layer thickness.

In case of a single layer over a half-space $n=2$ and hence we need to consider only one propagator matrix. Therefore,

$$
\begin{aligned}
& A_{11}=\cos Q_{1}, \\
& A_{21}=i\left(\mu_{1} \gamma_{1}\right)^{-1} \sin Q_{1}
\end{aligned}
$$


and

$$
f^{0}=f^{I}\left(\frac{2}{\cos Q_{1}+b \sin Q_{1}}\right),
$$

where

$$
b=\frac{\mu_{1} \gamma_{1}}{\mu_{2} \gamma_{2}} .
$$

To examine our code, we reproduced the amplification of the planar SH-waves as shown in Haskell's paper [1] as a function of incidence angle and period for the average crustal model. The model properties are crustal density $\rho_{1}=2.869$ $\mathrm{g} / \mathrm{cm} 3$, crustal shear wave velocity $\beta_{1}=3635 \mathrm{~m} / \mathrm{sec}$, crustal thickness $h_{1}=$ $37000 \mathrm{~m}$, mantle density $\rho_{2}=3.337 \mathrm{~g} / \mathrm{cm} 3$ and mantle shear wave velocity $\beta_{2}$ $=4600 \mathrm{~m} / \mathrm{sec}$. The reproduction amplification factor is shown in Figure 2 .

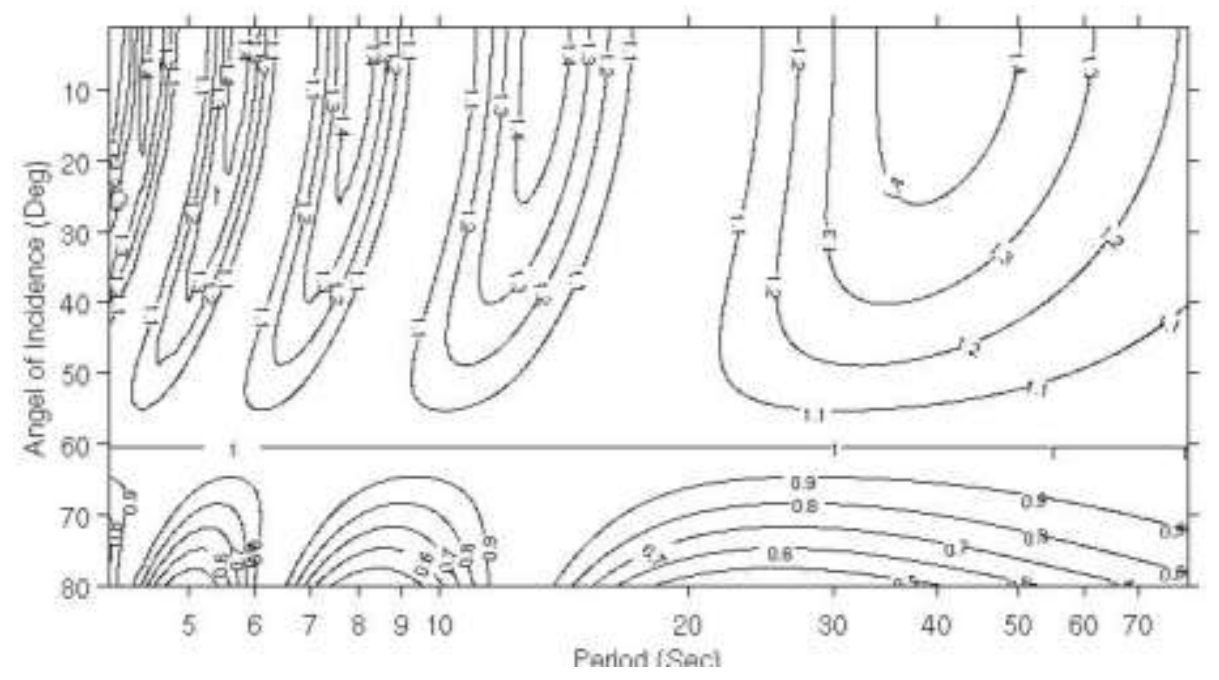

Figure 2 Reproduction of amplification of SH-waves to half-space average crustal model from Haskell [1].

\section{Application to the Bandung Basin Structure}

Using microtremor array data, the 1-D S-wave velocity structure at each observation point can be obtained by inversion of the dispersion curve. The result of microtremor data processing for the Bandung basin has been reported by Satake and Harjono [6]. By interpolating all 1-D structures and constraining the edge basin (zero depth), the 3-D velocity structure can be obtained. The 
interfaces between two layers are shown in Figure 3. The structure at the western part of the basin is not so representative because the observation point coverage is not good. More survey points are needed to resolve that area.
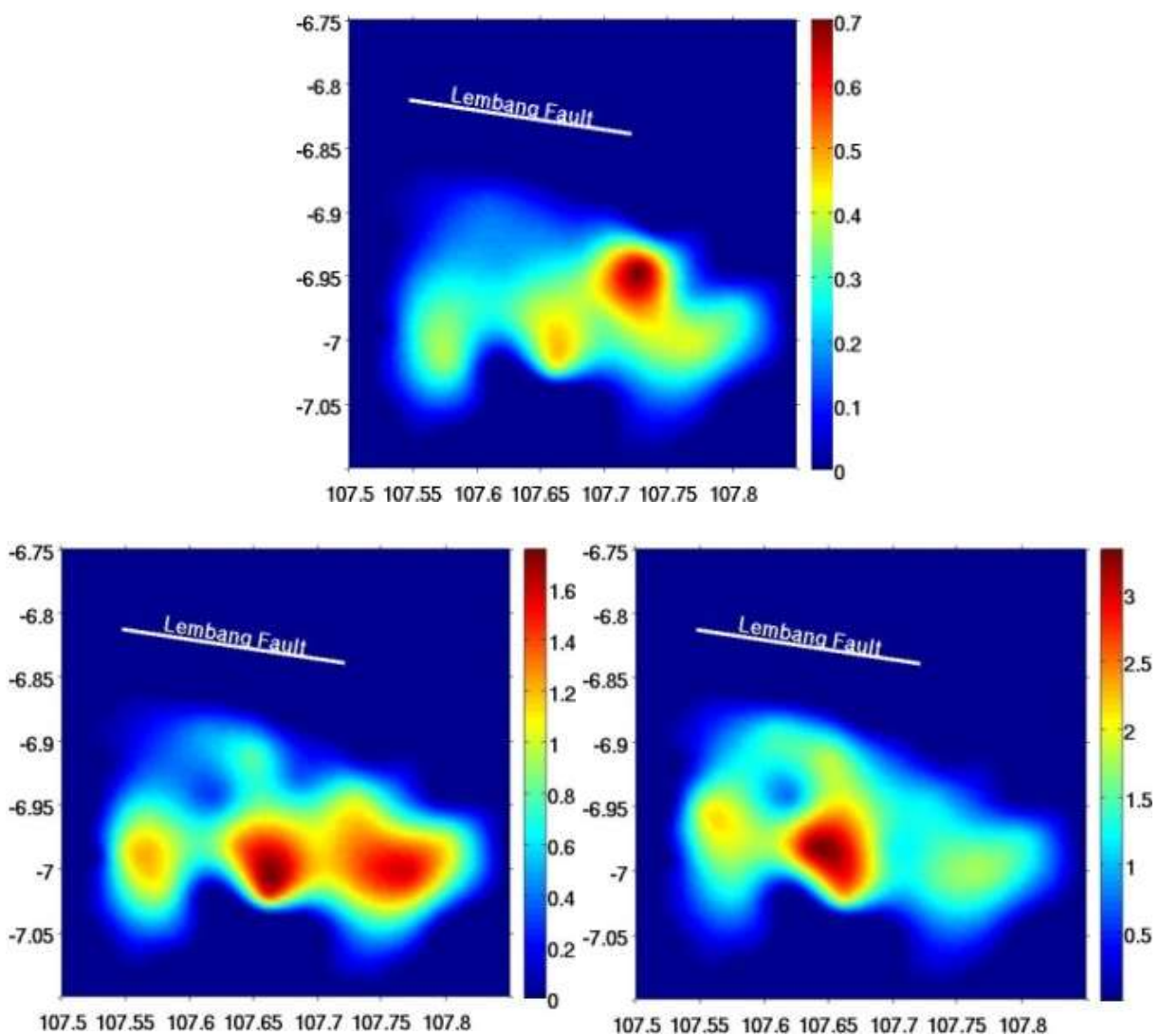

Figure 3 The interfaces of boundary of top sediment layer (top), middle sediment layer (left-bottom), and bottom sediment layer (right-bottom). The color bar of each interface indicates the depth in kilometers.

As can be seen from the above formulation, the elastic properties are needed to calculate the amplification. Layer properties obtained from microtremor array observation were used in this research, as shown in Figure 4. The thickness of each sediment layers was determined for all interfaces, as shown in Figure 3.

Beside that, the amplification factor is also dependent on the incident angle and period or frequency. We assume one fault plane of the Lembang fault with one asperity at the center of the plane predicted from Irikura and Miyake [1]. The hypocenter is placed at the center-base of the asperity, as shown in Figure 5. Therefore, the incident angle at the top of the basement is easy to estimate from 
the homogeneous basement layer. We use the minimum dominant frequency of $0.3 \mathrm{~Hz}$ and the maximum one of $1.5 \mathrm{~Hz}$ obtained from a microtremor survey of Bandung City by Marjiyono and Afnimar [7].

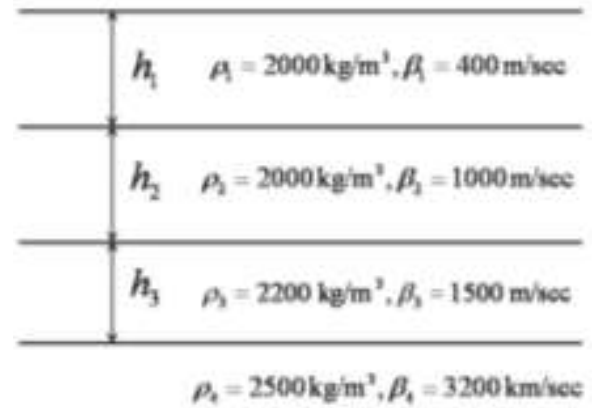

Figure 4 Half-space model of each observation position. The elastic properties were obtained from microtremor array observation and the thicknesses of the sediment layers were interpolated also from microtremor array observation.

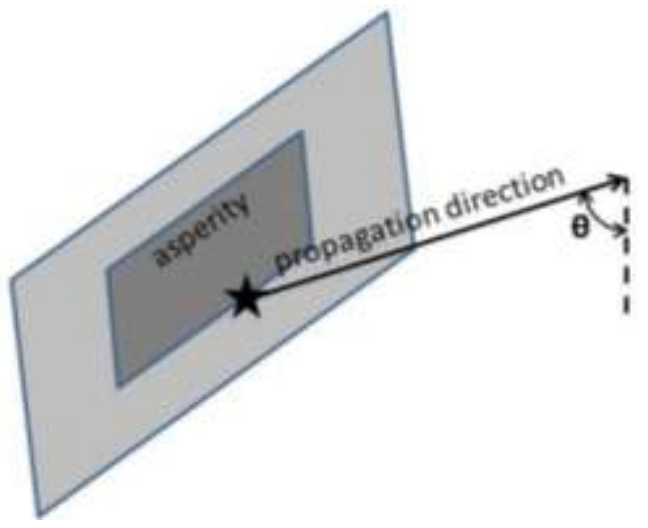

Figure 5 Schematic of wave propagation from the Lembang fault. The star symbol is the hypocenter point and $\theta$ is the incident angle at the basement top.

The estimation of the amplification factor for a multilayer model of the Bandung basin is shown in Figure 6 for frequency $0.3 \mathrm{~Hz}$ and in Figure 7 for frequency $1.5 \mathrm{~Hz}$. The amplification factor for frequency $0.3 \mathrm{~Hz}$ shows the degradation values with respect to the distance from the center of the Lembang fault, whereas there are many smaller amplification spots occurring periodically for frequency $1.5 \mathrm{~Hz}$. This pattern can be analyzed by calculating the amplification at one point as a function of incident angle and frequency. We calculated it at a point $(6.93 \mathrm{~S}, 107.57 \mathrm{E})$ where the depth of the basement is $1.5324 \mathrm{~km}$, the top of the third sediment layer is $0.4685 \mathrm{~km}$, and the top of the second sediment layer is $0.1617 \mathrm{~km}$. The amplifications at that point are shown 
in Figure 8. With the same value of incidence angle, the value of amplification for frequency $0.3 \mathrm{~Hz}$ is higher than its value for frequency $1.5 \mathrm{~Hz}$. The analysis for other points is the same as for point $(6.93 \mathrm{~S}, 107.57 \mathrm{E})$.

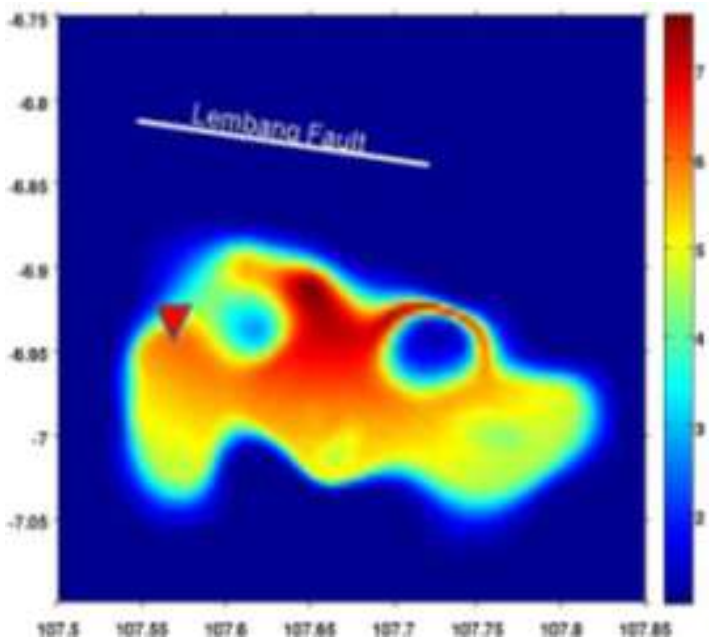

Figure 6 Amplification estimation for multilayer model of the Bandung basin for frequency $0.3 \mathrm{~Hz}$. The color bar indicates the amplification value. The red triangle symbol indicates the point where the amplification analysis in Figure 8 was made.

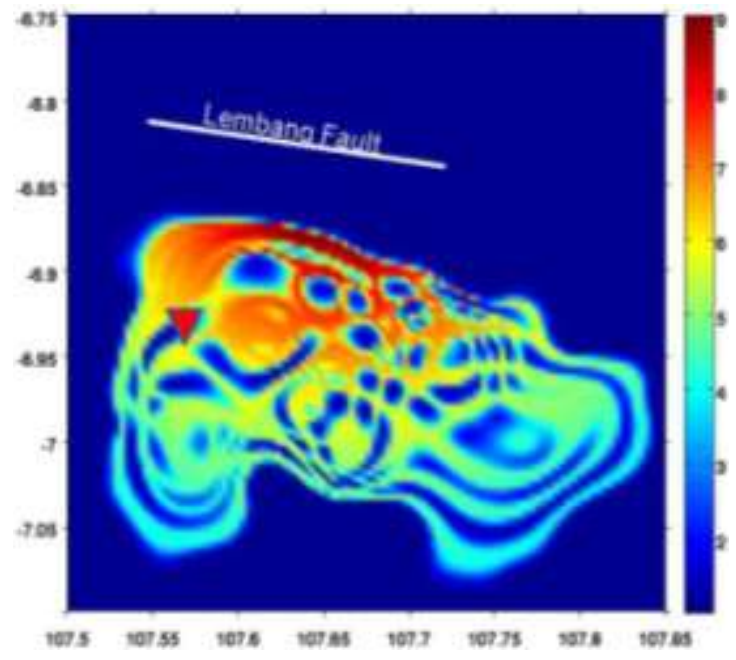

Figure 7 Amplification estimation for multilayer model of the Bandung basin for frequency $1.5 \mathrm{~Hz}$. The color bar indicates the amplification value. The red triangle symbol indicates the point where the amplification analysis in Figure 8 was made. 
The amplification of $0.3 \mathrm{~Hz}$ displays a simpler pattern than that of $1.5 \mathrm{~Hz}$ except for two areas at the northern part of the basin (indicated by two blue-colored areas), whose low values are related to the thin sediment layer. These low values are consistent with the general pattern that the amplification will be larger if the sediment layer is thicker, and vice versa. The amplification of these areas for frequency $1.5 \mathrm{~Hz}$ has a complicated pattern. This phenomenon is caused by dependence of the amplification not only the thickness of the sediment but also on the incidence angle and the frequency.

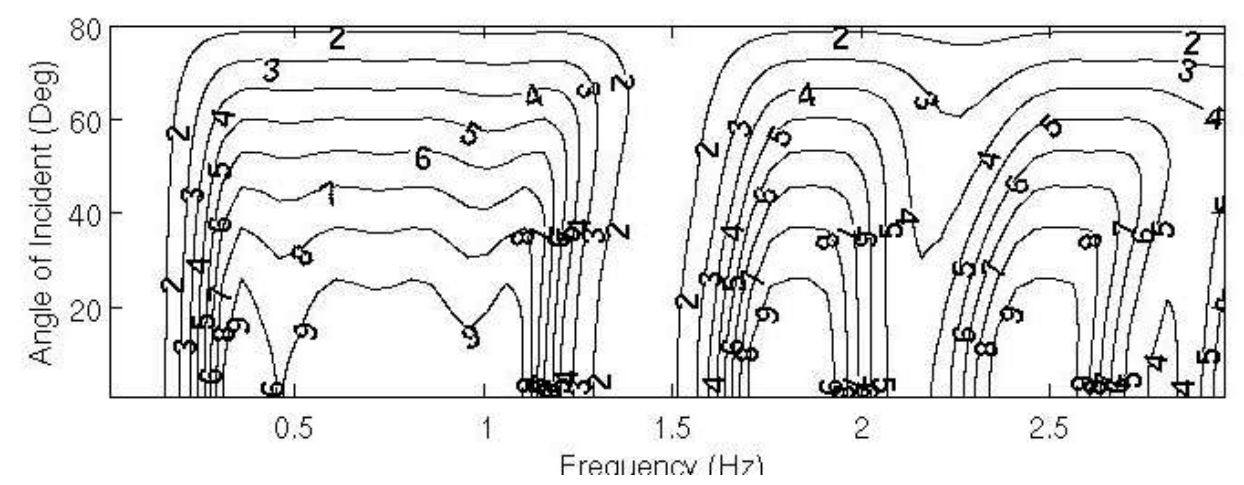

Figure 8 Amplification estimation at a point (107.57 E, $6.93 \mathrm{~S})$, indicated by triangles in Figures 6 and 7, in the Bandung basin as a function of angle of incidence and frequency.

\section{Discussion}

We have estimated the amplification of SH-waves in the Bandung basin area. This amplification map can be used as an additional consideration for decision makers to draw up a building code for this area. According to the results above, the amplification is dependent on frequency. Furthermore, the dominant frequency value varies with the variation of the sediment structure. Therefore an exploration of the dominant frequency should be conducted for this area using a method such as microtremor observation. Apart from that, the sediment structure is still smooth because of small number of observation points in the microtremor array observation. In the future, this should be improved by using microtremor array observation or background noise observation. These methods are better suited for populated areas because artificial sources are not needed. That way we will get not only the sediment structure but also the shear wave velocity, which are necessary in civil engineering.

We note here that this amplification estimation should be revised in order to involve the undulation of the interfaces and shallowest structures of the Bandung basin. Their influence on the amplification has been investigated by 
Semblat, et al. [8], who found that the increase of amplification and duration by 2-D basin effects compares to the 1-D model.

\section{$5 \quad$ Conclusions}

The synthetic classical amplification factor for the Bandung basin has been estimated. The amplification values increase by mean northward closer to the source position. This is a serious problem because the northern part is a densely populated area. The dominant frequency is another parameter that must be considered because it can change the amplification value drastically.

\section{References}

[1] Irikura, K. \& H. Miyake, Recipe for Predicting Strong Ground Motion from Crustal Earthquake Scenarios, Pure Appl. Geophys., 168, pp. 85104, 2010.

[2] Haskell, N.A., Crustal Reflection of Plane SH-Waves, J. Geophys. Res., 65, pp. 4147-4150, 1960.

[3] Lumbanbatu, U., Morphotectonic Relation to Tectonic Activity in Bandung Basin and Surrounding Area (Text in Indonesian, unpublished paper), Geology Survey Center, 2007.

[4] Thomson, W.T., Transmission of Elastic Waves through a Stratified Solid Medium, J. Appl. Phys., 21, pp.89-93, 1950.

[5] Haskell, N.A., The Dispersion of Surface Waves on Multilayered Media, Bull. Seism. Soc. Am., 43, pp. 17-34, 1953.

[6] Satake, K. \& Harjono, H., Multi-Disciplinary Hazard Reduction from Earthquake and Volcanoes in Indonesia, Final Report, JST-JICARISTEK-LIPI, pp. 165, 2012.

[7] Marjiono \& Afnimar, Earthquake Hazard Microzonation in The Bandung City Based on Mictrotremor Data (Text in Indonesian with English abstract), Journal of Geological Resources, 21, pp. 41-49, 2011.

[8] Semblat, J.F., Kham, M., Parara, E., Bard, P.Y., Pitilakis, K., Makra, K. \& Raptakis, D., Seismic Wave Amplification: Basin Geometry vs Soil Layering, Soil Dynamics and Earthquake Engineering, 25, pp. 529-538, 2005. 